\title{
Decision-to-delivery intervals and total duration of surgery for Caesarean sections in a tertiary general hospital
}

Tuck Chin Tiffany Wong ${ }^{1}$, MBBS, Chang Qi Hester $\underline{\text { Lau }}^{1}$, MBBS, Eng Loy $\underline{\text { Tan }}^{2}$, MMed, MRCoG, Devendra Kanagalingam ${ }^{2}$, MBBS, FRCOG

\begin{abstract}
INTRODUCTION This study aimed to determine the decision-to-delivery intervals (DDIs), total duration of surgery and factors influencing these for Caesarean sections (CSs).

METHODS A retrospective study was conducted of all CSs performed from August 2013 to June 2014 at a single tertiary general hospital. Data collected included maternal demographics, indications for CS, category of urgency, DDI, total duration of surgery, grade of first surgeon and number of previous CSs.

RESULTS In total, 488 CSs (Category 1: $n=28$; Category 2: $n=137$; Category 3: $n=184$; Category 4: $n=139$ ) were studied. Overall mean duration of surgery was 41.7 minutes. Mean DDI was 23.9 minutes and 64.5 minutes for Category 1 and Category 2 CSs, respectively. For Category 1 CSs, deliveries during office hours had a significantly shorter DDI than deliveries out of office hours $(p<0.05)$. For Category 2 CSs, deliveries during office hours had a significantly longer DDI $(p<0.05)$. Total duration of surgery for senior surgeons was significantly shorter than for trainee surgeons $(p<0.05)$. Women with no previous CSs had a significantly shorter duration of surgery than those who had one or more $(p<0.05)$. CONCLUSION The majority of the deliveries were within the recommended DDI corresponding to the degree of urgency of CS. The influence of time of day on DDI might be due to challenges of time taken to transfer patients to operating theatres. Total duration of surgery was influenced by surgical experience, history of previous CS and individual surgical styles and preferences.
\end{abstract}

Keywords: Caesarean section, decision-to-delivery interval, fetal distress, total duration of surgery

\section{INTRODUCTION}

In 2010, the Royal College of Obstetricians and Gynaecologists (RCOG) standardised the classification of urgency of Caesarean sections (CSs) into four defined categories, ${ }^{(1)}$ which was modified from Lucas et al. ${ }^{(2)}$ The RCOG classification categorises CSs according to whether there is maternal or fetal compromise: Category 1 comprises cases with immediate threat to life of woman or fetus; Category 2 comprises cases with fetal or maternal compromise with no immediate threat to life; Category 3 comprises cases without fetal or maternal compromise but requiring early delivery; and Category 4 comprises non-urgent or elective deliveries that can be timed to suit the woman and maternity services (Table I). This was a change from the traditional classification of CSs as 'emergency and elective', as the traditional classification is of limited value for the audit of obstetric and anaesthetic outcomes, and the spectrum of urgency that occurs in obstetrics is lost within a single 'emergency' category. ${ }^{(2)}$ With the new classification, more meaningful comparisons and audit of obstetric and anaesthetic practices as well as maternal and fetal outcomes are possible.

Historically, the standard adopted was to achieve a decisionto-delivery interval (DDI) of 30 minutes for emergency CS. However, this 30-minute rule has not been directly supported by clinical trials or experimental evidence. Hence, in the 2012 edition of Guidelines for Perinatal Care, published jointly by the
American College of Obstetricians and Gynecologists and the American Academy of Pediatrics, it was agreed that hospitals should have the capability of accomplishing delivery within 30 minutes, but that this timing should take into consideration maternal and fetal risks and benefits, and hence the DDI should be acceptable based on local circumstances and logistics. ${ }^{(3)}$ Thus, the National Institute for Health and Care Excellence guidelines recommended that the 30-minute rule be used as an audit target for capabilities rather than as a strict requirement for Category 1 deliveries, and that both 30 minutes and 75 minutes be considered as targets for Category 2 deliveries. ${ }^{(4)}$ However, a systemic review and meta-analysis by Tolcher et al concluded that delivery within 30 minutes was not achieved in a substantial proportion of cases. ${ }^{(5)}$

This study aimed to perform an audit of the DDIs and total duration of CSs performed at a tertiary general hospital to compare our timings against the recommended DDIs, with an aim to assess local standards and look at factors that might affect the total duration of surgery or DDIs. The total duration of CSs has not been extensively studied and we were interested in establishing institutional norms as well as look at factors that would influence this parameter.

\section{METHODS}

We conducted a retrospective study of consecutive CSs performed at Singapore General Hospital, Singapore, from August 2013 to

${ }^{1}$ Department of Obstetrics and Gynaecology, KK Women's and Children's Hospital, ${ }^{2}$ Department of Obstetrics and Gynaecology, Singapore General Hospital, Singapore Correspondence: Dr Tiffany Wong, Medical Officer, Department of Obstetrics and Gynecology, KK Women's and Children's Hospital, 100 Bukit Timah Road, Singapore 229899. tiffany.wong@mohh.com.sg 
Table I. Classification of Caesarean sections based on the degree of urgency and presence or absence of maternal or fetal compromise.

\begin{tabular}{ll}
\hline Urgency & Definition \\
\hline Maternal/fetal compromise & Immediate threat to life of woman or fetus \\
No maternal/fetal compromise & No immediate threat to life of woman or fetus \\
& Requires early delivery \\
\hline
\end{tabular}

Table II. Total duration of surgery and decision-to-delivery interval (DDI) for each category of urgency of Caesarean section.

\begin{tabular}{|c|c|c|c|c|c|c|c|}
\hline \multirow[t]{2}{*}{ Urgency } & \multirow[t]{2}{*}{ No. of patients } & \multicolumn{3}{|c|}{ Total duration of surgery (min) } & \multicolumn{3}{|c|}{ DDI (min) } \\
\hline & & Mean & Median & Range & Mean & Median & Range \\
\hline Category 1 & 28 & 39.4 & 37.5 & $20.0-65.0$ & 23.9 & 24.5 & $8.0-45.0$ \\
\hline Category 2 & 137 & 39.2 & 40.0 & $20.0-100.0$ & 64.5 & 50.0 & $15.0-745.0$ \\
\hline Category 3 & 184 & 40.8 & 40.0 & $20.0-95.0$ & - & - & - \\
\hline Category 4 & 139 & 45.7 & 40.0 & $20.0-255.0$ & - & - & - \\
\hline
\end{tabular}

June 2014. There were 490 patients for whom CS was performed during the study period. We excluded two women whose case records had no operating theatre pro forma, as the pro forma provided information on the time of decision-making, time of transfer to operating theatre, time of delivery and the category of urgency of CS. A total of 488 patients for whom CS was performed was thus analysed. We did not study DDI for Category 3 and Category $4 \mathrm{CSs}$, as no recommended target DDI exists for these groups of non-urgent deliveries.

Information collected included age of the mother, gravidity and parity, gestational age, singleton or multiple gestations, indications for CS, category of urgency of CS, time of decisionmaking, time of transfer to operating theatre, time of delivery, DDI, total duration of surgery, grade of first surgeon for the operation, and number of previous CSs. Neonatal outcomes were also collected, which included Apgar scores at one minute and five minutes, neonatal deaths, and the level of care ward that the neonate was admitted to, i.e. neonatal intensive care unit (NICU), special care nursery $(\mathrm{SCN})$ or general ward.

Total duration of surgery was taken to be the duration between first incision of the skin and placement of the last suture. DDI was taken to be the duration between the time the decision for a CS was made and the time of delivery of the baby. For women who had multiple gestations, the time of delivery was taken to be the time of the first baby's delivery. Surgeon grade was classified into two grades, namely senior surgeons (consisting of senior consultants, consultants and associate consultants) and trainee surgeons (consisting of registrars and residents). In our institution, senior surgeons have at least eight years of clinical experience in the speciality, are on the national specialist register and practise obstetrics independently. Trainee surgeons, by definition, are supervised in their clinical duties by senior surgeons. The time of delivery of the baby was classified into two groups: within office hours (0800-1700 hours) or out of office hours (1701-0759 hours).

The primary outcomes of the study were DDI and total duration of surgery. The DDIs were then used to compare against recommended standards as part of an audit and quality
Table III. Primary indications for Caesarean section in our cohort ( $n=488)$.

\begin{tabular}{lc}
\hline Indication & No. (\%) \\
\hline Previous Caesarean section(s) & $113(23.2)$ \\
Cephalopelvic disproportion or poor progress of & $107(21.9)$ \\
labour & \\
Fetal factors (e.g. non-reassuring fetal status) & $72(14.8)$ \\
Malpresentation (e.g. breech) & $61(12.5)$ \\
Multiple pregnancies & $27(5.5)$ \\
Antepartum haemorrhage & $26(5.3)$ \\
Hypertensive disorders & $19(3.9)$ \\
Maternal medical conditions & $9(1.8)$ \\
Maternal request & $28(5.7)$ \\
Others & $26(5.3)$ \\
\hline
\end{tabular}

improvement process. The data was analysed using IBM SPSS Statistics version 20.0 (IBM Corp, Armonk, NY, USA) and MannWhitney $U$ test for statistical analysis. Statistical significance was fixed at $p<0.05$.

\section{RESULTS}

The mean total duration of surgery for the 488 patients in our study cohort was 41.7 minutes and the mean DDI for all Category 1 and Category 2 CSs was 57.6 minutes. Our audit showed that the mean DDI for Category 1 CSs was 23.9 minutes (Table II) and 23 (82.1\%) deliveries were done within the recommended timing of 30 minutes. For Category 2 CSs, the mean DDI was 64.5 minutes and 119 (86.9\%) deliveries were within the recommended timing of 75 minutes. Among the primary indications for CS across all categories, the top indications were previous CS, cephalopelvic disproportion or poor progress of labour, fetal factors and malpresentation (Table III).

The mean and median DDIs of senior surgeons and trainee surgeons for Category 1 and Category 2 CSs are shown in Table IV. There were no significant differences in DDIs for senior surgeons and trainee surgeons for both Category 1 and Category 2 CSs when analysed separately. Between different grades of surgeons across 


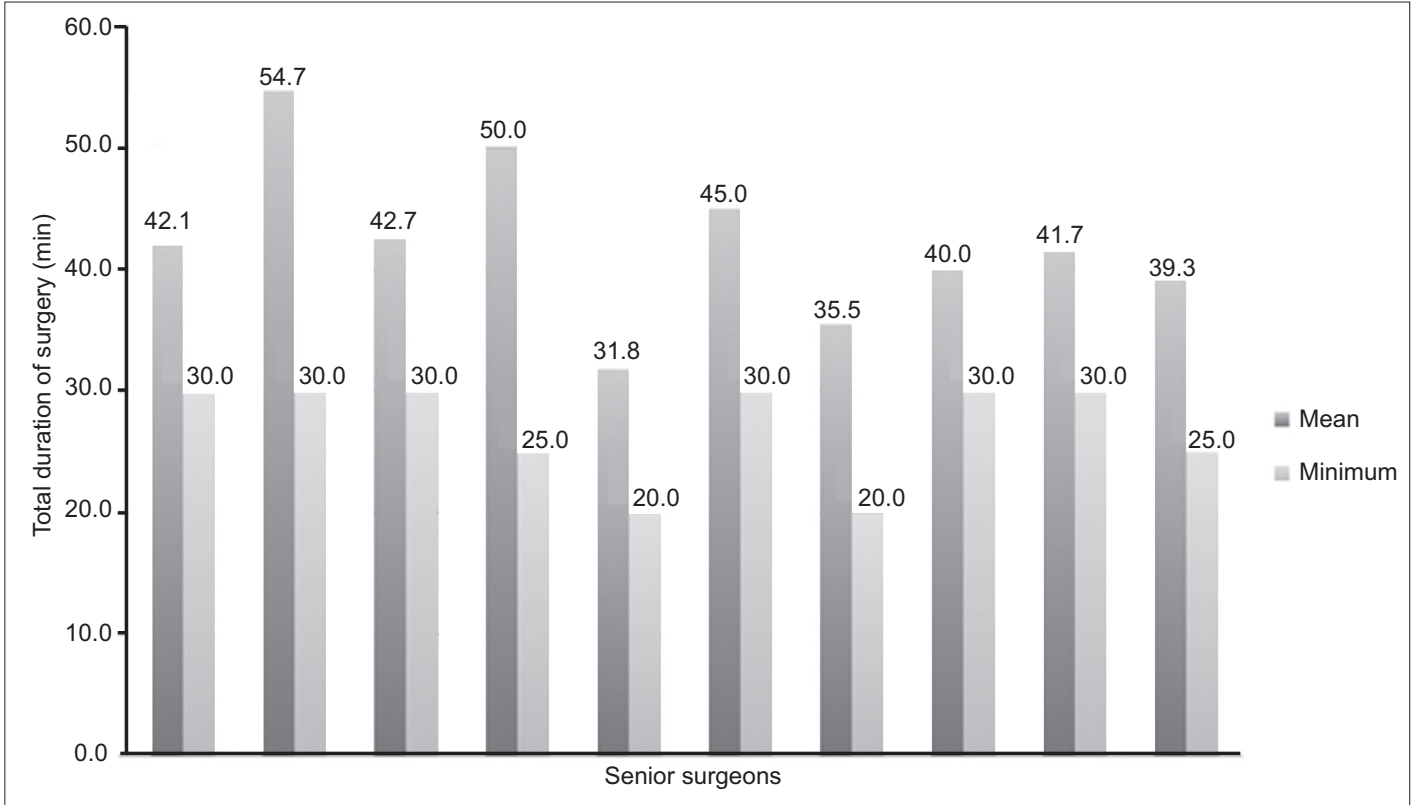

Fig. 1 Graph shows the mean and minimum total durations of surgery of each of the ten senior surgeons who had completed $\geq 10$ Caesarean sections during the study period.

Table IV. Decision-to-delivery interval (DDI) for different grades of surgeons for Category 1 and 2 Caesarean sections.

\begin{tabular}{llccccc}
\hline Urgency & Grade of surgeon & No. of patients & \multicolumn{2}{c}{ DDI (min) } & \multicolumn{2}{c}{ p-value } \\
\cline { 4 - 6 } & & & Meange & Median & $8.0-34.0$ & 0.280 \\
\hline Category 1 & Senior & 18 & 22.4 & 22.5 & $10.0-45.0$ & \\
& Trainee & 10 & 26.7 & 27.0 & $15.0-745.0$ & 0.747 \\
\multirow{2}{*}{ Category 2} & Senior & 100 & 62.7 & 49.0 & $23.0-577.0$ \\
& Trainee & 37 & 69.4 & 52.0 & \\
\hline
\end{tabular}

Senior surgeons comprise senior consultants, consultants and associate consultants; trainee surgeons comprise registrars and residents.

Table V. Total duration of surgery for different grades of surgeons across all categories of urgency.

\begin{tabular}{lcccc}
\hline $\begin{array}{l}\text { Grade of } \\
\text { surgeon }\end{array}$ & $\begin{array}{c}\text { No. of } \\
\text { patients }\end{array}$ & \multicolumn{2}{c}{ p-value } \\
\cline { 2 - 4 } & Mean & 40.2 & Median & $20.0-255.0$ \\
\hline Senior & 388 & 47.4 & 37.5 & $<0.001^{*}$ \\
Trainee & 100 & 45.0 & $25.0-100.0$ \\
\hline
\end{tabular}

Senior surgeons comprise senior consultants, consultants and associate consultants; trainee surgeons comprise registrars and residents. * $\mathrm{p}<0.05$ was statistically significant.

all categories of urgency, the mean and median total durations of surgery are shown in Table $\mathrm{V}$. The duration of surgery for senior surgeons was significantly shorter than that for trainee surgeons $(p<0.05)$. The total duration of surgery for each senior surgeon who had done $\geq 10$ CSs during the study period was analysed. The mean duration was 31.8-54.7 minutes, while the minimum duration was 20.0-30.0 minutes (Fig. 1).

The effects of time of day on DDI and duration of surgery were studied. The mean and median DDIs for Category 1 and Category 2 CSs performed during office hours and out of office hours are shown in Table VI. For Category 1 CSs, the DDIs for deliveries during office hours were significantly shorter than those for deliveries out of office hours $(p<0.05)$. For Category 2 CSs, the DDIs for deliveries during office hours were significantly longer than those for deliveries out of office hours $(p<0.05)$. The mean and median total durations of surgery for CSs performed during office hours and out of office hours across all categories of urgency are shown in Table VII. There were no significant differences in the duration of surgery for these two groups of patients.

The mean and median DDIs for Category 1 and Category 2 CSs in women with no previous CS and women with one or more previous CSs are shown in Table VIII. There were no significant differences in the DDIs for these two groups of women. The mean and median total durations of surgery of women with no previous CS and women with one or more previous CSs across all categories of urgency are shown in Table IX. Women with no previous CS had a significantly shorter total duration of surgery than women who had one or more CS $(p<0.05)$.

There were 453 singleton pregnancies, 34 twin pregnancies and 1 triple pregnancy in our study, making up a total of 524 neonates. There were two intrauterine deaths (IUD) but no neonatal deaths in our study. The neonatal outcomes (Apgar score at five minutes and level of care ward [NICU, SCN or general ward] that the neonate was sent to) for each category of urgency are shown in Table X. 
Table VI. Decision-to-delivery interval (DDI) for different times of the day on which the delivery occurred for Category 1 and 2 Caesarean sections.

\begin{tabular}{|c|c|c|c|c|c|c|}
\hline \multirow[t]{2}{*}{ Urgency } & \multirow[t]{2}{*}{ Time of day } & \multirow[t]{2}{*}{ No. of patients } & \multicolumn{3}{|c|}{ DDI (min) } & \multirow[t]{2}{*}{ p-value } \\
\hline & & & Mean & Median & Range & \\
\hline \multirow[t]{2}{*}{ Category 1} & Office hours & 12 & 20.0 & 22.0 & $8.0-30.0$ & $0.036^{*}$ \\
\hline & Out of office hours & 16 & 26.9 & 28.0 & $14.0-45.0$ & \\
\hline \multirow[t]{2}{*}{ Category 2} & Office hours & 42 & 98.9 & 58.5 & $15.0-745.0$ & $0.001^{*}$ \\
\hline & Out of office hours & 95 & 49.3 & 45.0 & $15.0-167.0$ & \\
\hline
\end{tabular}

Office hours: $0800-1700$ hours. Out of office hours: $1701-0759$ hours. ${ }^{*} p<0.05$ was statistically significant.

Table VII. Total duration of surgery for different times of the day on which the delivery occurred across all categories of urgency.

\begin{tabular}{lcccc}
\hline Time of day & No. of patients & \multicolumn{1}{c}{ p-value } & Total duration of surgery (min) \\
\cline { 3 - 4 } & & Mean & Median & $20.0-255.0$ \\
\hline Office hours & 302 & 42.9 & 40.0 & 0 \\
Out of office hours & 186 & 39.6 & 40.0 & $20.0-100.0$ \\
\hline
\end{tabular}

Office hours: 0800-1700 hours. Out of office hours: 1701-0759 hours.

Table VIII. Decision-to-delivery interval (DDI) among patients with previous Caesarean section(s) for Category 1 and 2 Caesarean sections.

\begin{tabular}{|c|c|c|c|c|c|c|}
\hline \multirow[t]{2}{*}{ Urgency } & \multirow[t]{2}{*}{ Previous Caesarean section(s) } & \multirow[t]{2}{*}{ No. of patients } & \multicolumn{3}{|c|}{ DDI (min) } & \multirow[t]{2}{*}{ p-value } \\
\hline & & & Mean & Median & Range & \\
\hline \multirow[t]{2}{*}{ Category 1} & None & 24 & 24.8 & 26.0 & $10.0-45.0$ & 0.263 \\
\hline & $\geq 1$ & 4 & 19.0 & 19.5 & $8.0-29.0$ & \\
\hline \multirow[t]{2}{*}{ Category 2} & None & 110 & 58.4 & 48.0 & $15.0-577.0$ & 0.082 \\
\hline & $\geq 1$ & 27 & 89.2 & 55.0 & $15.0-745.0$ & \\
\hline
\end{tabular}

Table IX. Total duration of surgery among patients with history of previous Caesarean section(s) across all categories of urgency.

\begin{tabular}{lcccc}
\hline $\begin{array}{l}\text { Previous Caesarean } \\
\text { section(s) }\end{array}$ & No. of patients & \multicolumn{2}{c}{ p-value } \\
\cline { 3 - 4 } & & Mean & \multicolumn{2}{c}{ Medal duration of surgery (min) } \\
\hline None & 320 & 38.6 & 40.0 & $20.0-75.0$ \\
$\geq 1$ & 166 & 47.6 & 45.0 & $20.0-255.0$ \\
\hline
\end{tabular}

${ }^{*} \mathrm{p}<0.05$ was statistically significant.

Table X. Neonatal outcomes for each category of urgency of Caesarean section.

\begin{tabular}{|c|c|c|c|c|c|c|c|}
\hline \multirow[t]{3}{*}{ Urgency } & \multirow[t]{3}{*}{ Total } & \multicolumn{6}{|c|}{ No. (\%) } \\
\hline & & \multicolumn{3}{|c|}{ Apgar score at 5 min } & \multicolumn{3}{|c|}{ Disposition of neonate } \\
\hline & & $7-10$ & $4-6$ & $0-3$ & IUD & NICU or SCN & General ward \\
\hline Category 1 & 29 & $24(82.8)$ & $5(17.2)$ & $0(0.0)$ & $0(0.0)$ & $8(27.6)$ & $21(72.4)$ \\
\hline Category 2 & 144 & $134(93.0)$ & $9(6.3)$ & $1(0.7)$ & $1(0.7)$ & $23(16.0)$ & $120(83.3)$ \\
\hline Category 3 & 196 & 194 (99.0) & $0(0.0)$ & $2(1.0)$ & $1(0.5)$ & $28(14.3)$ & $167(85.2)$ \\
\hline
\end{tabular}

IUD: intrauterine death; NICU: neonatal intensive care unit; SCN: special care nursery

\section{DISCUSSION}

Our audit showed that for Category $1 \mathrm{CSs}$, the mean DDI was 23.9 minutes and $82.1 \%$ deliveries were done within the recommended time period of 30 minutes. For Category 2 CSs, the mean DDI was 64.5 minutes and $86.9 \%$ deliveries were performed within the recommended time period of 75 minutes. When combined for both categories, the mean DDI was 57.6 minutes. The review and meta-analysis of 34 studies by Tolcher et al showed that $79.0 \%$ of Category 1 deliveries and $36.0 \%$ of Category 2 deliveries were achieved within 30 minutes. They also found that the mean decision-to-incision or delivery recorded in 27 studies were 21.2 minutes, 42.6 minutes and 32.4 minutes for Category 1 CSs, Category 2 CSs, and combined Categories 1 and 2 CSs, respectively. ${ }^{(5)}$ Our results were comparable with these findings.

In our study, CSs that exceeded the recommended timings were due to delays in transferring the patient to the operating theatre. This is because our centre is a multidisciplinary centre with heavy demand for operating theatres and anaesthetic manpower. To reduce DDI times at our hospital, we have implemented a strict emergency CS protocol. In addition, improvements have been made to the layout of the labour ward (i.e. removal of a wall and addition of a door) to allow direct access to the operating theatre. 
Local data from another tertiary hospital in Singapore has previously been published. Kwek et al reported an overall mean DDI of 14.6 (range 3-32) minutes. ${ }^{(6)}$ Nair et al reported a mean DDI of crash CSs of 10.8 (range 3-31) minutes. This was made possible by ensuring that there is always an operating theatre on standby throughout the day for crash CSs and by activating a 'code green' via a public address system, to activate the team required instead of notifying individual doctors. Each team member would be required to know his or her own roles and respond appropriately. ${ }^{(7)}$ We are aware of the option of using the public address system and are looking into suitable modification of its use for our unit.

Lim et al reported a mean DDI of 7.7 minutes and also found that reasons for delay included transfer from the delivery suite to the operating theatre and anaesthetic reasons. ${ }^{(9)}$ These were overcome by implementing a strict protocol, having efficient labour ward and operating theatre layouts, and ensuring that there was sufficient trained manpower throughout the day. The hospital also had a practice of preparing all patients for general anaesthesia by pre-oxygenating them immediately when they arrived in the theatre, so that general anaesthesia could be initiated immediately, if needed. ${ }^{(9)}$

Internationally, similar reasons have been found to be the cause of delay. Spencer and MacLennan conducted a study among hospitals with differing levels of facilities and found that the delay was due to a lack of staff in Level 1 hospitals, lack of theatres in Level 2 hospitals and anaesthetic complications in Level 3 hospitals. ${ }^{(10)}$ Sayegh et al, in a study conducted in a French maternal hospital, found that DDIs were mainly influenced by the time taken to get the patient into the operating theatre, as the mean decision-to-operating theatre interval accounted for $45.6 \%$ of the mean DDI for emergency and urgent CSs. The authors concluded that improving communication within the perinatal team could reduce the decision-to-operating theatre interval. ${ }^{(11)}$

We also recognise that, even within the predefined categories of urgency, there is a difference in the true level of urgency for delivery in each case. For example, in Category 1 CSs, cord prolapse and uterine rupture are acute emergencies in which immediate delivery is mandatory and delay may result in neonatal morbidity or mortality. Conversely, delivery within 30 minutes is usually advocated for a failed attempt at operative vaginal delivery with forceps or vacuum extractor, but in these circumstances, a delay is less likely to cause harm if fetal heart rate monitoring is satisfactory. It is possible that obstetricians also take this into account when performing urgent CSs. Unnecessarily rushed CSs may be at risk of anaesthetic and surgical complications, such as visceral injury, due to haste.

In our study, senior surgeons had a greater maximum duration of surgery compared to trainee surgeons. This could be because the senior surgeons were likely to have been involved in potentially more difficult operations, which would have accounted for some surgeries taking over three hours. Among senior surgeons, the mean duration of surgery ranged from 31.8 minutes to 54.7 minutes. This range could be attributable to teaching, as some surgeons choose to supervise residents during procedures, or it may be a reflection of the different surgical styles and preferences of surgeons.

Previous studies by MacKenzie and Cooke ${ }^{(12)}$ and Livermore and Cochrane ${ }^{(13)}$ noted that the time of day did not influence DDIs. However, these studies were conducted prior to the introduction of a classification for urgency of CSs, which was used in our study. This would mean that CSs that are currently classified as Category 1 and Category 2 may have been analysed together in earlier studies. Our study further analysed Category 1 and Category 2 CSs separately, and found that for Category 1 CSs, deliveries during office hours had a significantly shorter median DDI than deliveries out of office hours. We postulate that during office hours, there is greater availability of obstetrics and anaesthetic staff, who can be activated immediately once it is confirmed to be an emergency procedure, hence shortening DDIs. However, for Category 2 CSs, deliveries during office hours had a significantly longer DDI than deliveries out of office hours. We postulate that this was because the demand for operating theatre space was greater during the day when elective operating lists are in progress. This is especially so in the setting of a multidisciplinary tertiary general hospital, such as our centre. As the urgency of Category 2 CSs is more discretionary, this often results in Category 2 cases being slotted in when an operating theatre becomes available, provided that fetal and maternal well-being is not compromised.

Women who had one or more previous CSs had a longer median total duration of surgery than those who had none previously. As expected, this was most likely related to CSs being more surgically demanding due to intra-abdominal adhesions from previous CSs. This information would be of value for women considering a maternal-request Caesarean or vaginal birth after CS and could help them make an informed choice.

There has been controversy regarding the 30-minute standard for CS deliveries. Tolcher et al's systemic review and meta-analysis of 13 studies, which reported neonatal outcomes for Category 1 deliveries accomplished within 30 minutes vs. those that took more than 30 minutes, showed that neonatal outcomes did not differ by delivery interval. ${ }^{(5)}$ Hence, the significance of failing to keep to the 30-minute standard for CS deliveries is uncertain. It is important to recognise that the DDI does not measure the obstetrician's response time to intrapartum emergency. In cases that are more severe and irreversible, such as placental abruption, cord prolapse and uterine rupture, obstetricians tend to act more promptly, so the decision to have a CS for such patients would be made sooner. However, in less severe and reversible cases, the obstetrician may choose to observe or undertake other corrective measures before finally making the decision to perform a CS. As these variables are not reflected in the DDI, insult-to-delivery time (i.e. the time between the occurrence of the suspected peripartum insult and time of delivery) should perhaps be used as an alternative measure. Leung and Lao found that, although all studies had investigated perinatal outcome with DDI, the onset of fetal hypoxia does not start at the time of decision but around the time of onset of fetal bradycardia. ${ }^{(14)}$ Thus, if the analysis was limited to DDI instead 
of insult-to-delivery, any association between perinatal outcomes and the duration of fetal hypoxia would remain masked. ${ }^{(14)}$ However, there are no established standards on the insult-todelivery timing, and such a parameter is likely to vary significantly depending on the severity of the insult.

In conclusion, the majority of women undergoing Category 1 and Category 2 CSs at our centre had deliveries within the recommended DDIs corresponding to the degree of urgency of their CSs. The influence of the time of day on DDI in our study might be due to challenges related to the time taken to transfer the patients to the operating theatre. Total duration of surgery was influenced by surgical experience and history of previous CSs. Individual surgical styles and preferences also influenced the total duration of surgery, as evidenced by the wide variation among surgeons of similar seniority.

\section{REFERENCES}

1. Royal College of Obstetricians \& Gynaecologists, the Royal College of Anaesthetists. Classification of urgency of caesarean section - a continuum of risk (Good Practice No. 11). Available at: https://www.rcog.org.uk/globalassets/ documents/guidelines/goodpractice11classificationofurgency.pdf. Accessed May 19, 2016.

2. Lucas DN, Yentis SM, Kinsella SM, et al. Urgency of caesarean section: a new classification. J R Soc Med 2000; 93:346-50.

3. The AAP Committee on Fetus and Newborn, ACOG Committee on Obstetric Practice. Guidelines for Perinatal Care. 7th ed. American Academy of Pediatricians, 2012: 192.

4. National Institute for Health and Care Excellence. Caesarean section: NICE guidelines [CG132] 2011. Available at: https://www.nice.org.uk/guidance/ cg132. Accessed May 19, 2016.

5. Tolcher MC, Johnson RL, El-Nashar SA, West CP. Decision-to-incision time and neonatal outcomes: a systemic review and meta-analysis. Obstet Gynecol 2014; 123:536-48.

6. Kwek K, Yeap ML, Tan KH, Tee JC, Yeo GS. Crash caesarean section--decisionto-delivery interval. Acta Obstet Gynecol Scand 2005; 84:914-5.

7. Nair S, Mandang S, Siraj SH, Kwek K, Tan KH. A Review of Crash Caesarean Sections at KK Women's and Children's Hospital, Singapore in 2006. Singapore J Obstet Gynaecol 2012; 43:23-6.

8. Wee HY, Quek SC. Delivery by caesarean section. Effective system of mobilisation is used in Singapore. BMJ 2001; 323:931.

9. Lim Y, Shah MK, Tan HM. Evaluation of surgical and anaesthesia response times for crash caesarean sections--an audit of a Singapore hospital. Ann Acad Med Singapore 2005; 34:606-10.

10. Spencer MK, MacLennan AH. How long does it take to deliver a baby by emergency Caesarean section? Aust N Z J Obstet Gynaecol 2001; 41:7-11.

11. Sayegh I, Dupuis O, Clement HJ, Rudigoz RC. Evaluating the decision--to-delivery interval in emergency caesarean sections. Eur J Obstet Gynecol Reprod Biol 2004; 116:28-33.

12. MacKenzie IZ, Cooke I. What is a reasonable time from decision-to-delivery by caesarean section? Evidence from 415 deliveries. BJOG 2002; 109:498-504.

13. Livermore LJ, Cochrane RM. Decision to delivery interval: a retrospective study of 1,000 emergency caesarean sections. J Obstet Gynaecol 2006; 26:307-10.

14. Leung TY, Lao TT. Timing of caesarean section according to urgency. Best Pract Res Clin Obstet Gynaecol 2013; 27:251-67. 